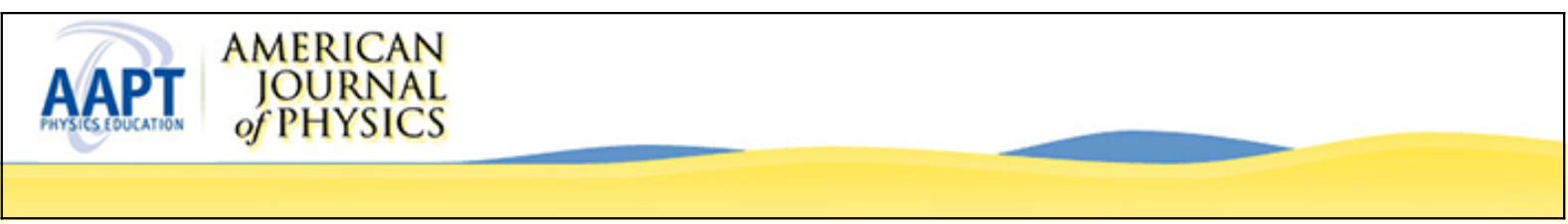

\title{
Apparatus for investigating resonance with application to magnetic resonance imaging
}

Sytil Murphy, Dyan L. Jones, Josh Gross, and Dean Zollman

Citation: American Journal of Physics 83, 942 (2015); doi: 10.1119/1.4928177

View online: http://dx.doi.org/10.1119/1.4928177

View Table of Contents: http://scitation.aip.org/content/aapt/journal/ajp/83/11?ver=pdfcov

Published by the American Association of Physics Teachers

\section{Articles you may be interested in}

A Modification to Maxwell's Needle Apparatus

Phys. Teach. 53, 282 (2015); 10.1119/1.4917434

Resonance in a head massager

Phys. Teach. 53, 245 (2015); 10.1119/1.4914570

A New Direction: How a Compass Pointed the Way to Clearing Up an Attractive Misconception Phys. Teach. 50, 398 (2012); 10.1119/1.4752040

Exploring Magnetic Fields with a Compass

Phys. Teach. 49, 45 (2011); 10.1119/1.3527756

Reversing Compass Needles

Phys. Teach. 41, 434 (2003); 10.1119/1.1616487

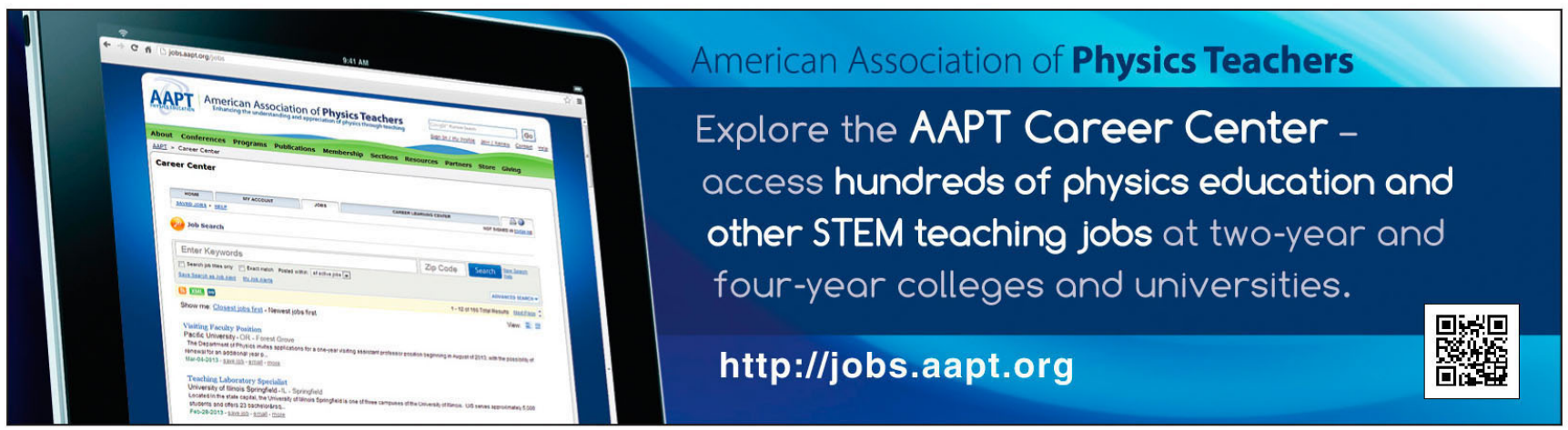




\title{
Apparatus for investigating resonance with application to magnetic resonance imaging
}

\author{
Sytil Murphy \\ Dyan L. Jones \\ Department of Physics, Mercyhurst University, Erie, Pennsylvania 16546 \\ Josh Gross $^{\text {a) }}$ and Dean Zollman \\ Department of Physics, Kansas State University, Manhattan, Kansas 66506-2601
}

Institute of Environmental and Physical Science, Shepherd University, Shepherdstown, West Virginia 25443

(Received 2 April 2014; accepted 27 July 2015)

\begin{abstract}
Resonance is typically studied in the context of either a pendulum or a mass on a spring. We have developed an apparatus that enables beginning students to investigate resonant behavior of changing magnetic fields, in addition to the properties of the magnetic field due to a wire and the superposition of magnetic fields. In this resonant system, a compass oscillates at a frequency determined by the compass's physical properties and an external magnetic field. While the analysis is mathematically similar to that of the pendulum, this apparatus has an advantage that the magnetic field is easily controlled, while it is difficult to control the strength of gravity. This apparatus has been incorporated into a teaching module on magnetic resonance imaging. @ 2015 American Association of Physics Teachers.

[http://dx.doi.org/10.1119/1.4928177]
\end{abstract}

\section{INTRODUCTION}

In introductory courses, students can be frequently motivated by studying physics in the context of biology and medicine. Medical imaging provides an especially rich context for studying physics principles. Magnetism and resonance are at the core of the physics associated with nuclear magnetic resonance (NMR) and its application to magnetic resonance imaging (MRI). An undamped compass oscillating in an external magnetic field allows for the exploration of magnetic resonance and can be easily related to NMR. This paper describes a single apparatus that can be used to study the physics of a current-carrying wire, resonance in the context of magnetism, and its use in a learning activity on NMR/MRI.

Many experiments and apparatus guides illustrate portions of the physics of a current-carrying wire. For example, Sutton $^{1}$ describes a method for building a dip magnet for looking at one component of the Earth's magnetic field, while Meiners et al. $^{2}$ suggest constructing a tangent galvanometer to show the direction of the magnetic field. In another work, Meiners ${ }^{3}$ uses a series of fixed compass needles and iron filings to show the circular nature of the magnetic field created by a current-carrying conductor. Both PSSC physics ${ }^{4}$ and Genova ${ }^{5}$ describe an experiment using a compass to investigate the magnetic field due to a currentcarrying wire similar to what we describe below.

Resonance is typically introduced in the context of a pendulum, a mass-on-a-spring, or an RLC circuit. ${ }^{6}$ NMR can be introduced using a variety of magnetic systems. Hill describes an apparatus for demonstrating magnetic resonance of a small bar magnet in a static magnetic field with an oscillating field (provided by an audio-frequency generator) at right angles to the static field. ${ }^{7}$ This apparatus is rather complex and does not lend itself to the simple study of electromagnetism. Connors and Al-Shamali ${ }^{8}$ considered the oscillation of dipoles in the Earth's magnetic field. However, because there is no convenient way of adding energy to their system after it is initially set into oscillation, such a system would not be useful for demonstrating resonance.
Section II of this paper discusses the construction of our apparatus. This section is followed by a description of how the apparatus can be used to probe the field due to a wire (including the field's direction), superposition of the wire's field with the Earth's field, and the measurement of the decrease in the wire's field strength as a function of the height above the wire. Section IV discusses the use of this apparatus to study resonance, including the measurement of the resonance frequency as a function of the proximity of a bar magnet. The connection between the physics studied with this apparatus and NMR and MRI is the topic of Sec. V.

\section{THE APPARATUS}

This simple apparatus is composed of a wire running in a groove cut into a transparent acrylic sheet as shown in Fig. 1. The wire's path is approximately a right triangle, each leg of which measures $25.4 \mathrm{~cm}$. The wire is connected to a switch and a battery. When the switch is closed, current flows in the circuit and creates a magnetic field, which will cause a compass placed near the wire to deflect in the direction of the field. If one of the two legs of the triangle is aligned perpendicular to Earth's magnetic field and current applied, a compass placed over it will not deflect, because the horizontal part of the magnetic field due to the wire is parallel to that of Earth, which the compass is already aligned with. However, near the other leg, which is parallel to the horizontal component of Earth's field, the wire's field adds vectorially to that of Earth and causes a deflection as discussed in detail below. In addition to these static scenarios, tapping the switch near a particular frequency can drive the compass needle into resonance.

Clear acrylic allows compasses placed both above and below the apparatus to be easily viewed. The materials for this apparatus are reasonably inexpensive, costing about $\$ 25$ per apparatus, excluding the compasses; undamped compasses can be obtained for approximately $\$ 10$ per dozen. The same phenomena can be observed using a version of the apparatus made of foam core board instead of clear acrylic. This version is even less expensive and easy to make, 


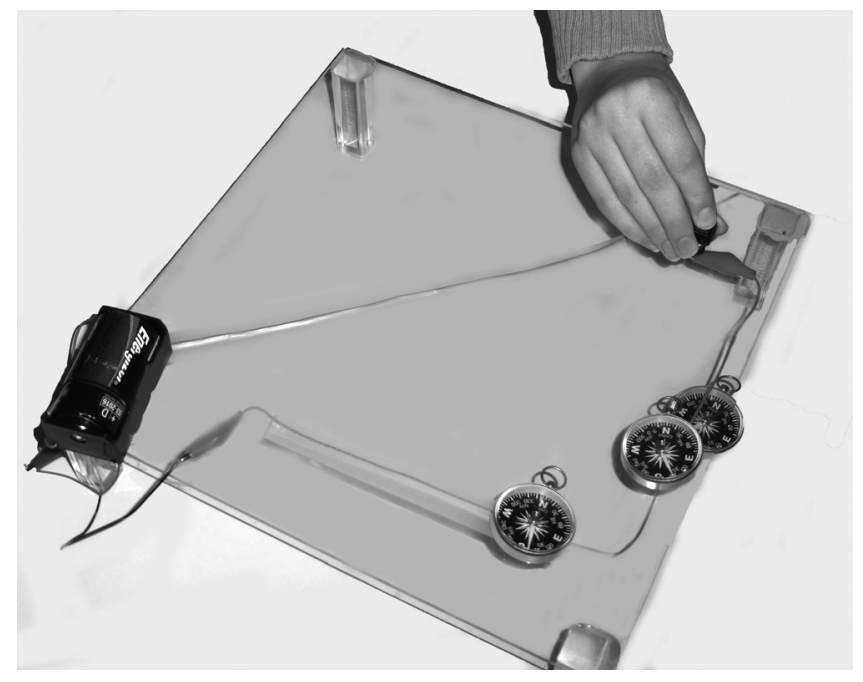

Fig. 1. Photograph of the apparatus, including three compasses placed above or below the wire with the circuit closed.

requiring only a ruler and a razor blade to cut the groove, but has the disadvantages of being opaque and not as pleasing to the eye. The opacity has been overcome by cutting a window in the foam core for viewing a compass placed below it.

\section{INVESTIGATION OF THE MAGNETIC FIELD DUE TO A CURRENT-CARRYING WIRE}

\section{A. Studying the field's direction}

The magnetic field direction indicated by the compass when the circuit is closed is the superposition of the field due to the Earth and the field due to the wire. Students can determine many properties of the magnetic field due to the wire by observing how compasses behave when the circuit is closed. For example, if one wire is oriented North/South such that a nearby compass points parallel to the wire when the circuit is open, then the observed deflection of a compass below the wire is in the opposite direction of a compass above the wire, as shown by the upper two compasses in Figs. 1 and 2. Since one side of the triangle is oriented North/South, parallel to the horizontal component of the Earth's magnetic field, then the other side is aligned East/ West. A compass placed either above or below the East/ West wire will not deflect when the circuit is closed, as shown by the lowest compass in Figs. 1 and 2. The knowledge that torque is zero when the force and the moment arm are parallel (or in this case when the magnetic field due to the wire and the magnetic moment of the compass are parallel), coupled with these two observations, can lead to the conclusion that the magnetic field due to the wire is perpendicular to the wire and directed in opposite directions above and below. This observation was originally made by Oersted in $1820 .^{9}$

\section{B. Measurement of field strength as a function of distance}

The magnetic field $\vec{B}_{\text {wire }}$ due to a long wire is given in SI units by ${ }^{10,11}$

$$
\vec{B}_{\text {wire }}=\frac{\mu_{0} i}{2 \pi r} \hat{\varphi}
$$

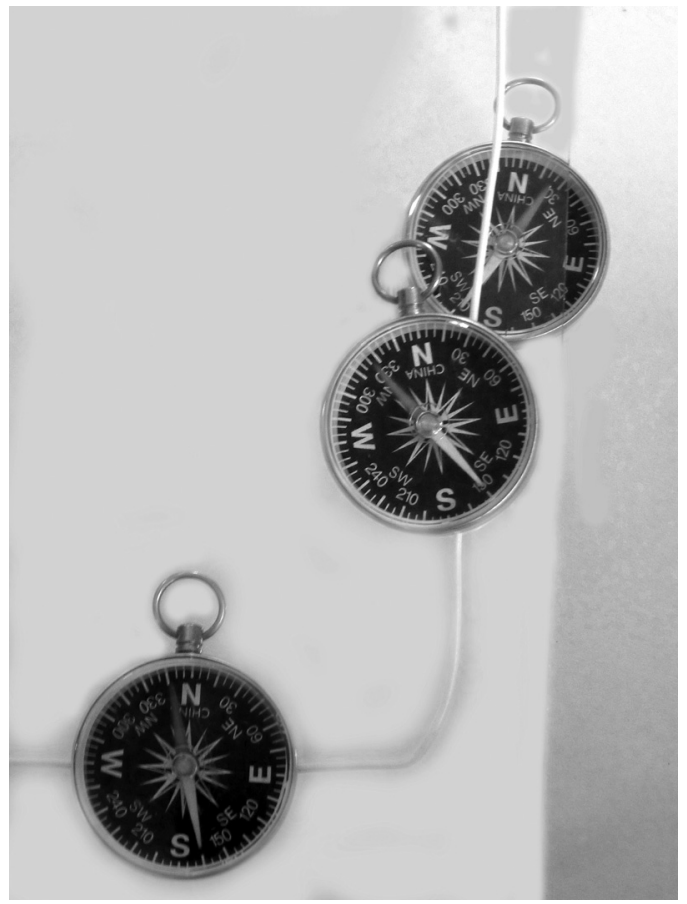

Fig. 2. Zoomed-in photo of the three compasses in Fig. 1. The lowest compass does not deflect because the torque on the needle is zero. The other two compasses deflect in opposite directions because they are on opposite sides of the wire (above and below).

where $\mu_{0}$ is the permeability of free space, $i$ is the current in the wire, $r$ is the distance from the wire, and $\hat{\varphi}$ is the unit vector in the azimuthal direction around the wire. Because the field due to the wire depends on the distance from the wire, the deflection of the compass needle decreases as the distance between a compass and the wire is increased. The measured value of the compass deflection angle can be compared with a theoretically calculated value by measuring the current in the circuit and the deflection angle when the compass is at various heights above the wire. The validity of the approximations used in this analysis is discussed in supplementary material (Supplement 1$).^{12}$

When the circuit is closed, the magnetic field experienced by the compass is a superposition of the wire's and the horizontal component of Earth's. The theoretical value of the deflection angle is calculated using the geometry shown in Fig. 3.

The deflection angle $\psi$ as a function of distance $r$ above the wire, for the total magnetic field is

$$
\psi(r)=\tan ^{-1}\left[\frac{B_{\text {wire }}(r)}{B_{\text {Earth }}}\right]=\tan ^{-1}\left[\frac{\mu_{0} i}{2 \pi r B_{\text {Earth }}}\right],
$$

where $B_{\text {wire }}$ and $B_{\text {Earth }}$ are the horizontal components of the wire's and Earth's fields, respectively. ${ }^{13,14} \mathrm{We}$ assume that the field due to the wire at the location of the compass is horizontal, so its horizontal component is the same as its magnitude. With the exception of the extreme highest and lowest points, good agreement between theory and data was achieved, as shown in Fig. 4. The error bars on the measured values are $\pm 2.5^{\circ}$ and represent the precision of the compass readings.

The disagreement for the first data point in Fig. 4 is due to a breakdown in the assumption about the direction of the magnetic field due to the wire. At the lowest point, the 


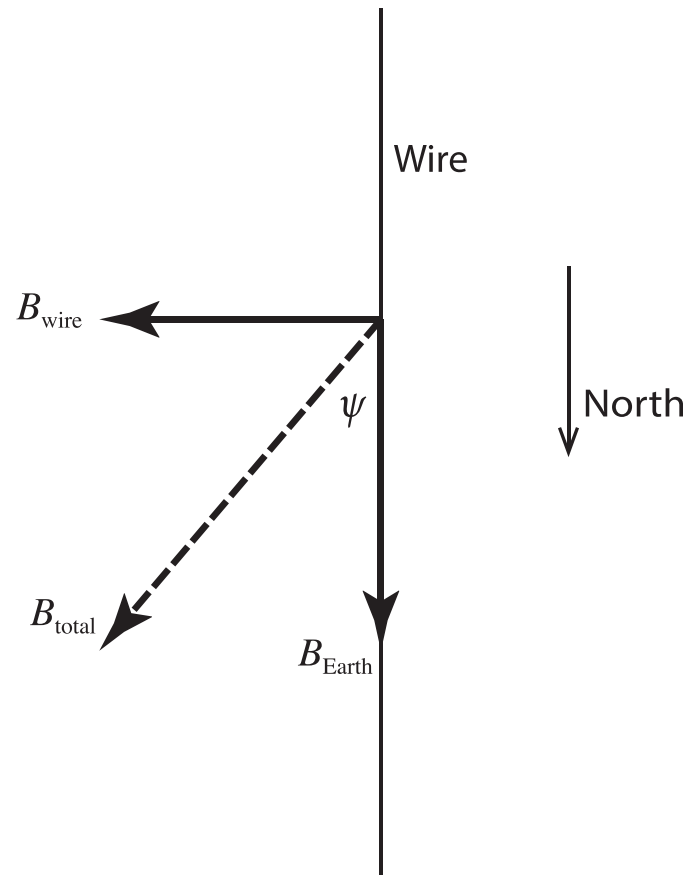

Fig. 3. Geometry for calculating the dependence of the total magnetic field on the distance above the wire.

variation in the horizontal component of the wire's magnetic field becomes important. As shown in Fig. 5, the magnetic field at the lowest point varies significantly along the length of the compass. For comparison, the variation of the field's strength at $2 \mathrm{~cm}$ is also shown. The calculations leading to Fig. 5 are given in supplementary material (Supplement 2). ${ }^{12}$

\section{OSCILLATORY BEHAVIOR}

The two key components of a resonant system are an oscillator and a mechanism for driving the oscillator at its natural frequency. A simple pendulum, for example, oscillates at a natural frequency that depends upon its length $i$ and the gravitational field strength $g$ and can be driven by providing a "kick" in the direction of the motion during each swing. In our magnetic system, the compass's natural frequency

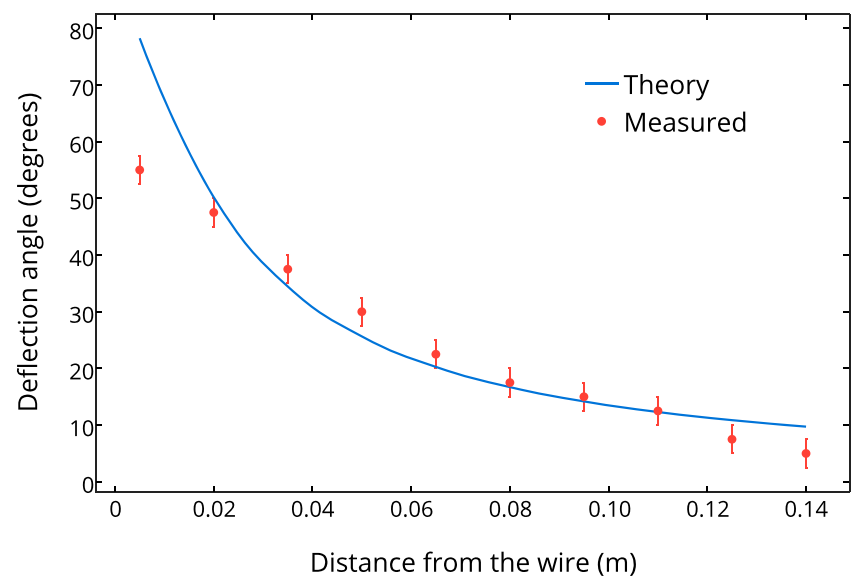

Fig. 4. Measured and theoretical deflection angle (measured from north) for a compass above a current-carrying wire as a function of the distance from the wire. The error bars on the measurements are $\pm 2.5^{\circ}$, the precision of the compass readings.

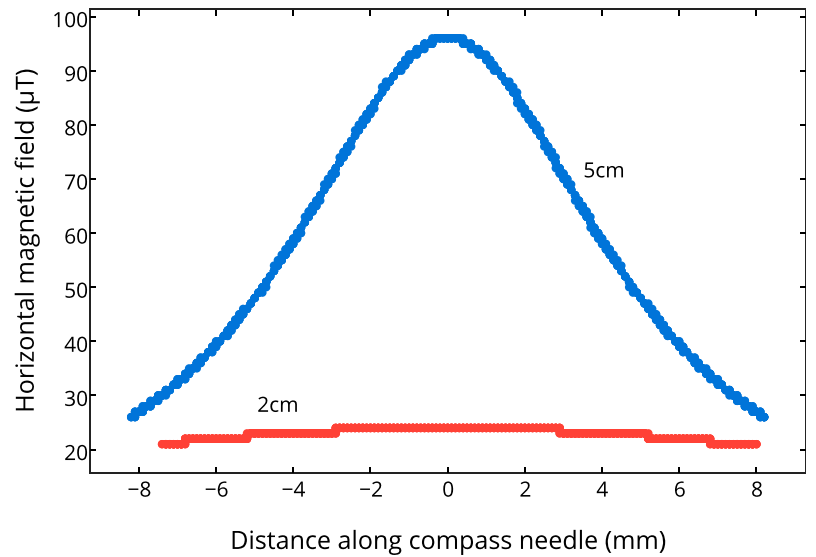

Fig. 5. The strength of the horizontal $(y)$ component of the magnetic field due to the wire at heights of $5 \mathrm{~mm}$ and $2 \mathrm{~cm}$ above the wire, as a function of the distance along the projection of a compass needle placed above the wire. The deflection angle is assumed to be the measured value of $55^{\circ}$ at $5 \mathrm{~mm}$ and $47.5^{\circ}$ at $2 \mathrm{~cm}$, and the current was measured at $2.4 \mathrm{~A}$.

depends on both the physical and magnetic properties of the compass needle as well as the strength of the external magnetic field provided by either the Earth or a bar magnet. Energy can be added to the system by tapping the switch at the compass's natural frequency.

\section{A. A compass as a resonant system}

Mathematically, the pendulum and oscillating compass are analogous. The torque $\tau$ on a simple pendulum is

$$
\tau=I \alpha=-m l g \sin \theta
$$

where $I$ is the pendulum's moment of inertia, $\alpha$ is its angular acceleration, $m$ is its mass, and $\theta$ is its angle relative to vertical. Typically one plugs in $I=m l^{2}$ and, after making the small-angle approximation, obtains the usual expression $\sqrt{g / l}$ for the angular frequency. However, the analogy to the compass is clearer if we retain $I$, writing the angular frequency as

$$
\omega=\sqrt{\frac{m l g}{I}} .
$$

Similarly, the torque on the compass needle is

$$
\tau=I \alpha=-\mu_{C} B \sin \theta
$$

where $I$ is the compass's moment of inertia, $\alpha$ is its angular acceleration, $\mu_{C}$ is its magnetic dipole moment, $B$ is the strength of the external magnetic field, and $\theta$ is the angle between the needle and the field. After making the smallangle approximation, we obtain for the angular oscillation frequency

$$
\omega=\sqrt{\frac{\mu_{C} B}{I}}
$$

an equation consistent with that published by Clack and Toepker. ${ }^{15}$

Equation (6) illustrates how the compass's oscillation frequency depends on the strength of the external magnetic field as well as on the compass's mechanical (I) and magnetic 
$\left(\mu_{C}\right)$ properties. When we exchanged a compass with a needle length of $\sim 0.03 \mathrm{~m}$ for one with a needle length of $\sim 0.01 \mathrm{~m}$, the oscillation frequency was observed to increase dramatically, consistent with the expected decrease in moment of inertia (although we cannot be sure that the magnetic dipole moment did not also change). For the experiment described below, the ratio $\mu_{c} / 1$ is determined and, therefore, it is not necessary to separate the two. If desired, one can estimate the needle's moment of inertia from its mass and length, and use this estimate to obtain an approximate value of $\mu_{c}$.

\section{B. A magnetic resonance experiment}

Exploring the dependence of the resonance frequency on the strength of the external magnetic field is easily accomplished by varying the distance between the compass and a bar magnet placed parallel to the wire, as shown in Fig. 6. In this respect, the compass resonance has an advantage over the pendulum because it is difficult to control the strength of the gravitational field-although some have done this by tilting the pendulum. ${ }^{16}$

There are (at least) two possible ways to measure the resonance frequency. An obvious choice is to have the students tap the switch at the resonance frequency (as shown in the supplemental online videos ${ }^{12}$ ). This is tactile and is useful in the same way as tapping on a pendulum. Students will come away with a definite impression of the functional dependence of the resonance frequency on the magnetic field. Thus, this approach is valuable pedagogically.

However, tapping the switch does not provide very accurate data, particularly at higher frequencies. ${ }^{17} \mathrm{~A}$ better alternative for data acquisition is to hold the switch down to give the compass an initial amplitude, wait for the compass to stop oscillating, and then release the switch. The compass then oscillates back and forth around its equilibrium position at the resonance frequency. This is analogous to pulling a pendulum back and then releasing it. As one would do with a pendulum, we timed several oscillations and then divided by the number of oscillations. The compasses are not frictionless and when the bar magnet is present and close to the compass the initial amplitude provided by the field due to the wire is small. Thus, the number of oscillations timed for each point varied but was as large as possible. The data are shown in Fig. 7.

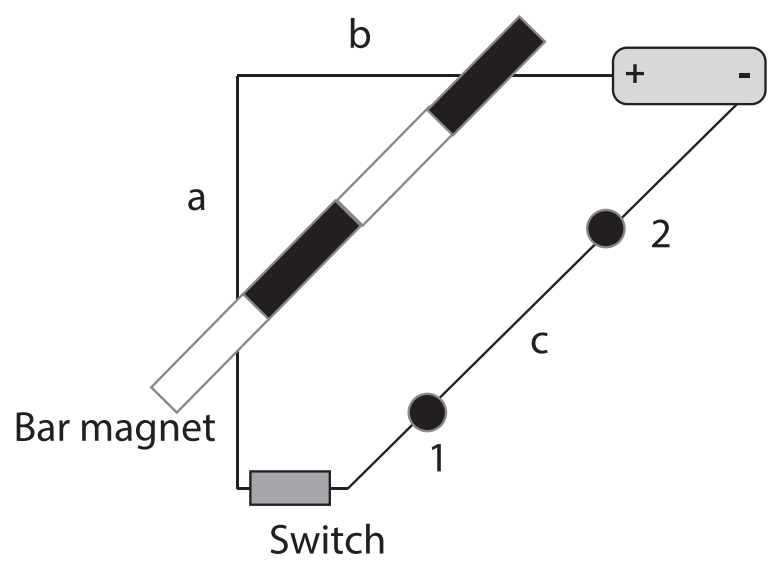

Fig. 6. Schematic of a hands-on activity that results in different resonance frequencies for the two compasses, 1 and 2, placed along wire $c$.

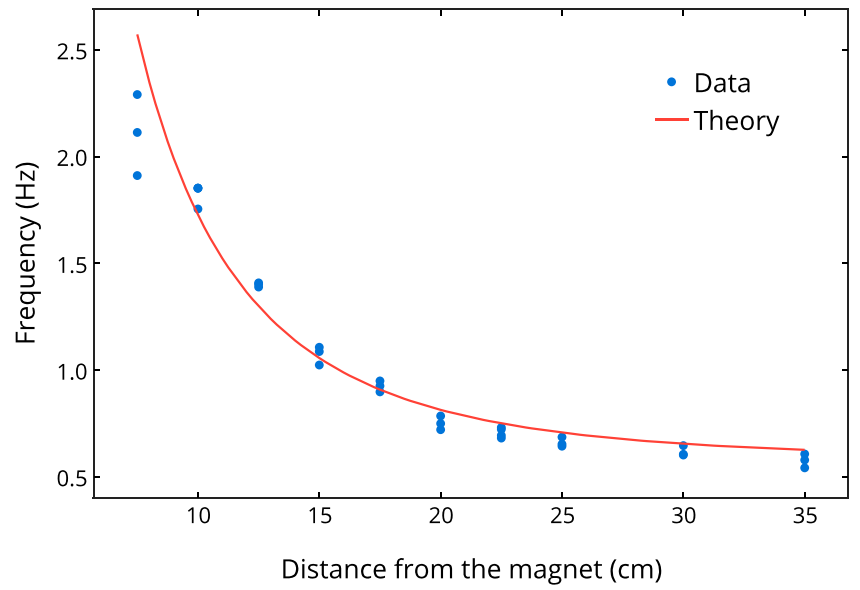

Fig. 7. Resonance frequency of a compass as a function of the separation between the compass and a bar magnet placed parallel to the wire. See the text for an explanation of the theoretical curve.

In order to calculate the theoretical curve shown in Fig. 7 , the bar magnet was approximated as a perfect magnetic dipole with a field given in cylindrical coordinates by

$$
\vec{B}_{\text {dipole }}(r, \varphi)=\frac{\mu_{0} \mu_{d}}{4 \pi r^{3}}[2 \cos (\varphi) \hat{r}+\sin (\varphi) \hat{\varphi}],
$$

where $\mu_{d}$ is the dipole moment of the magnet.

The field at the compass was assumed to be parallel to the wire below the compass $\left(\varphi=90^{\circ}\right)$, which is equivalent to ignoring any curvature of the field due to the bar magnet and initially aligning the wire with the Earth's field. The calculation has two unknowns: $\mu_{d}$ and the ratio $\mu_{c} / I$. Ignoring any breakdown in the small-angle approximation, $\mu_{c} / I$ can be determined using Eq. (6) once the resonance frequency in the absence of a bar magnet is measured. For our compass, this value was determined to be $6.53 \times 10^{5} \mathrm{~A} / \mathrm{kg}$.

When the bar magnet is present, the external magnetic field is the sum of Earth's field and the magnet's. We were careful to ascertain that the compass did not anti-align with Earth's field in order to align with the stronger bar magnet field. Thus, the magnitude of $B$ in Eq. (6) is given by

$$
B=B_{\text {Earth }}+B_{\text {dipole }}\left(r, \frac{\pi}{2}\right),
$$

where $B_{\text {Earth }}$ is the horizontal component of Earth's magnetic field. ${ }^{13}$ The value of $\mu_{d}$ was then adjusted to create the best fit between the theory and the data; for Fig. 7, we chose $\mu_{d}=1.6 \mathrm{Am}^{2}$.

At each separation distance in Fig. 7, the resonance frequency was measured at least three times, although the measured values were indistinguishable in most cases. The exception is the two sets of points with the smallest separation between the bar magnet and compass. The small size of the oscillator (compass needle) created a challenge in that systematic errors made it difficult to measure frequencies that were greater than about $2 \mathrm{~Hz}$. Thus, the disagreement between the theory and the experimental data at the smallest separation is most likely due to these experimental errors. However, another factor in the disagreement could be a breakdown in either of the assumptions in the theory: at that distance, the field due to the bar magnet may not be well approximated by that of a perfect dipole or the curvature of the bar magnet's field could be significant $\left(\varphi \neq 90^{\circ}\right)$. 


\section{APPLICATION TO UNDERSTANDING MRI}

The three basic components in both NMR and MRI are a collection of atoms of a particular type, a strong magnetic field that "aligns" the atoms, and an electromagnetic wave to add energy to the system. The difference between NMR and MRI is the inability of a basic NMR system to target a specific set of atoms, which MRI accomplishes by adding additional controls to the magnetic field. For both NMR and MRI, the resonance-or Larmor-frequency of the atoms is determined by the type of atom and the strength of the magnetic field. ${ }^{18}$ Our resonant system involving the compass also has three basic components: the compass, the external magnetic field to which the compass aligns, and the magnetic field due to the wire, which is used to add energy to the system. From Eq. (6), it can be seen that the compass's oscillation frequency depends on both the type of compass $\left(\mu_{\mathrm{c}}\right)$ and the strength of the external magnetic field $(B)$. In NMR and MRI, the resonance frequency of the atoms increases as the magnetic field is increased, just as the compass's oscillation frequency increases as the bar magnet is brought closer (see Fig. 7). Thus, the compass resonant system and the basic physics involved with NMR and MRI have a nice one-to-one correspondence.

We have used this system to create a learning activity on MRI as part of the Modern Miracle Medical Machines (MMMM) project at Kansas State University. ${ }^{19}$ The MMMM project aims to teach introductory students physics in the context of medical applications. Each activity developed as part of the MMMM project combines hands-on elements, computer-based visualizations, and a research-based pedagogy. As the MMMM materials are meant for introductory students without quantum mechanical backgrounds, they ignore or gloss over some of the specifics of the system being studied. An example in this project is that the MRI activity ignores the fact that Larmor precision is actually a circular oscillation rather than a back-and-forth oscillation through an equilibrium position like the compass needle.

The MRI activity begins with a study of the basics of magnetism, including the fact that a compass needle is a magnet that can be used to detect magnetic fields. The activity then moves into an investigation of the field due to a currentcarrying wire using the apparatus described here. The students observe that the compass deflects in opposite directions when placed above and below the wire. They also observe that the amount of deflection decreases as the compass's distance from the wire is increased. After this investigation of magnetism and the basics of electromagnetism, there is a short section describing the magnets used in MRI machines.

At this point, the students begin working with the concept of resonance. Because students are generally not familiar with a compass as a resonant system, we chose to begin studying resonance with a pendulum. After studying the pendulum, the students proceed to investigate the factors determining the resonance frequency of an oscillating compass.

We use the Simplified MRI PhET visualization ${ }^{20}$ to help students understand atomic resonance. To begin, connections are made between the elements of the resonant compass system and the elements of the PhET visualization using the NMR portion of the visualization. By switching back and forth between the two (hands-on and visualization), students gain an understanding that the resonance frequencies of both the compass and the atoms depend on the type of compass/ atom and the strength of the external field. The students are then confronted with trying to make only a portion of the "atoms" in the visualization resonate, as would be needed to actually create an image. After placing two compasses (as far apart as possible) along the same wire in the hands-on activity, the students are asked the corresponding question: how can they make only one compass resonate at a time? After considering this question, the students are instructed to place a pair of bar magnets at an angle to the wire, as shown in Fig. 6. Since the resonance frequency depends on the strength of the external magnetic field (which in this case is provided by both Earth and the bar magnets), the two compasses will now have different resonance frequencies. At this point, the students switch to the MRI portion of the visualization, observing that in addition to the original controls they had in the NMR portion, there are additional gradient field controls. The gradient fields have the same effect as the canted magnets in the hands-on portion and allow only a slice of the atoms to resonate. The students then look for differences in the resonance signals to locate a hidden tumor within the simulated patient.

\section{SUMMARY}

This paper describes the construction and use of a simple apparatus capable of probing the physics related to the magnetic field due to a wire, as well as providing a new context for the study of resonance. This apparatus provides a way to look at superposition of magnetic fields as well as for measuring the $1 / r$ dependence of the magnetic field. Studying resonance using this apparatus has the advantage that we can control the magnetic field, unlike the situation with a pendulum in which it is very difficult to control gravity. More importantly, this apparatus can be incorporated into a learning activity to teach some of the fundamental physics related to NMR and MRI. This activity uses the apparatus for learning about the magnetic field of a current-carrying wire as well as investigating the resonance of a compass.

\section{ACKNOWLEDGMENTS}

This work was supported in part by the National Science Foundation under Grant No. DUE 04-27645.

\footnotetext{
${ }^{a)}$ Present address: Department of Mathematics, Kansas State University, Manhattan, Kansas 66506.

${ }^{1}$ Demonstration Experiments in Physics, edited by Richard M. Sutton (McGraw-Hill, New York, 1938), p. 295.

${ }^{2}$ Harry F. Meiners, Walter Eppenstein, Ralph A. Olivia, and Thomas Shannon, Laboratory Physics, 2nd ed. (Wiley, New York, 1987), pp. 507-508.

${ }^{3}$ Harry F. Meiners, Physics Demonstrations Experiments (Ronald Press Company, New York, 1970), p. 921.

${ }^{4}$ Uri Haber-Schaim, John H. Dodge, Robert Gardner, and Edward A. Shore, PSSC Physics, 7th ed. (Kendall/Hunt, Dubuque, Iowa, 1991), p. 168.

${ }^{5}$ James J. Genova. "Magnetic fields of current carrying wires-An undergraduate laboratory experiment," Am. J. Phys. 40(5), 694-696 (1972).

${ }^{6}$ A. M. MacLeod. "Some demonstrations of resonance phenomena in mechanical systems," Phys. Educ. 4(5), 272-275 (1969).

${ }^{7}$ John J. Hill, "A magnetic resonance demonstration model," Am. J. Phys. 31(6), 446-449 (1963).

${ }^{8}$ Martin Connors and Farook Al-Shamali, "The magnetic torque oscillator and the magnetic piston," Phys. Teach. 45(7), 440-444 (2007).

${ }^{9}$ Bern Dibner, Oersted and the Discovery of Electromagnetism (Blaisdell, New York, 1962).

${ }^{10}$ Jeff Sanny and William Moebs, University Physics (Wm. C. Brown, Dubuque, 1996), p. 527.

${ }^{11}$ David Halliday, Robert Resnick, and Jearl Walker, Fundamentals of Physics, Extended, 8th ed. (Wiley, Hoboken, 2008), p. 767.
} 
${ }^{12}$ See supplementary material at http://dx.doi.org/10.1119/1.4928177 for Supplement 1 which discusses the validity of the assumptions made when calculating the theoretical magnetic field strength at the compass in Sec. III B. Supplement 2 gives the details of the theoretical calculations shown in Fig. 5. The third supplement is a collection of videos showing compasses being put into resonance by tapping the switch. The fourth supplement is the MRI activity instructions written as part of the Modern Miracle Medical Machines project at Kansas State University, which incorporates the apparatus discussed in this article.

${ }^{13} \mathrm{We}$ found that a $6-\mathrm{V}$ battery produced a magnetic field near the wire comparable to Earth's, giving deflections of about $45^{\circ}$. We tried placing a resistor into the circuit but found that even a small resistor drastically reduced the current.

${ }^{14}$ National Oceanic and Atmospheric Association, "Magnetic Field Calculators," < http://www.ngdc.noaa.gov/geomag-web/\#igrfwmm>. This web site gives the horizontal and vertical components as well as the total field at any location.

${ }^{15}$ Jhules A. M. Clack and Terrence P. Toepker. "Magnetic induction experiment," Phys. Teach. 28(4), 236-238 (1990).

${ }^{16}$ José Feliciano. "The variable gravity pendulum," Phys. Teach. 36(1), 51-52 (1998).

${ }^{17}$ Because of the low frequencies involved, using a function generator to drive the circuit was not possible with the equipment we had available.

${ }^{18}$ Roald K. Wangsness, Electromagnetic Fields, 2nd ed. (Wiley, New York, 1979), p. 556.

${ }^{19}$ The instructions for this activity are available in the online supplement for this article (Ref. 12) and also at the Modern Miracle Medical Machines web site, <http://web.phys.ksu.edu/mmmm/ $>$.

${ }^{20}$ The Simplified MRI visualization is available at the PhET Project web site, <http://phet.colorado.edu/en/simulation/mri $>$.

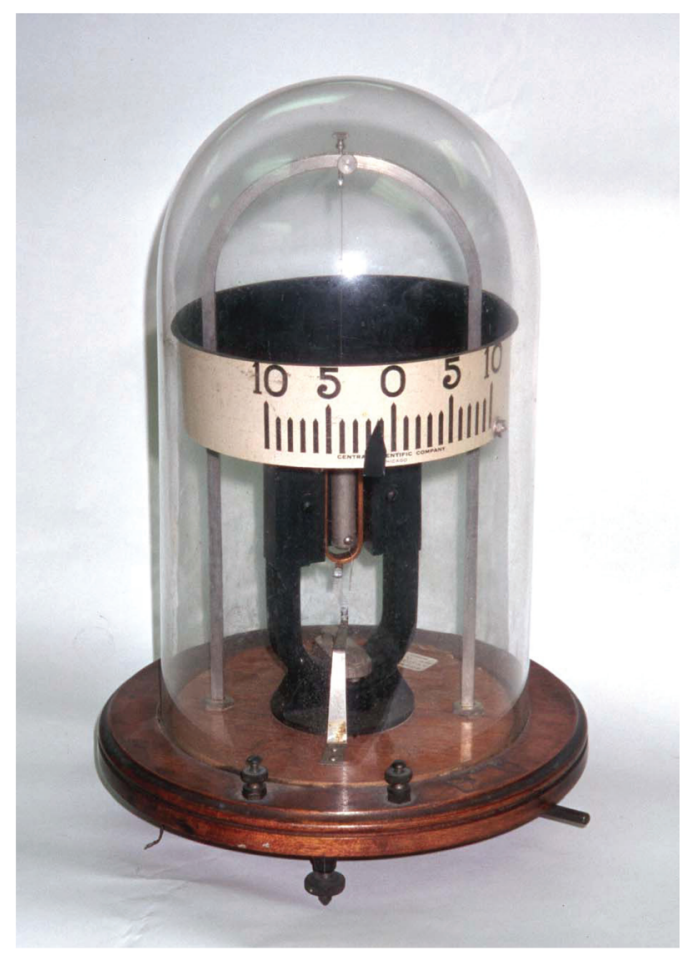

\section{Lecture-Table Galvanometer}

This lecture table galvanometer appears in the 1909 Central Scientific Company catalogue at a cost of $\$ 15.00$. Later catalogues describe it as "Originally designed after suggestions by Prof. R. A. Millikan [of the University of Chicago], with its open construction, large size scale and large pointer, is especially well adapted for use on the physics lecture table. The size of the scale divisions and figures, and the size and shape of the pointer makes it easy to read the deflections at a distance. A second scale and pointer provided on the back aid the teacher while doing classroom demonstrations. ... A deflection of one scale division $(6.1 \mathrm{~mm})$ on the circular scale $8.2 \mathrm{~cm}$ from the axis of the coil is produced by a current of 14 microamperes." This instrument, which has a 1911 patent date on it, is in the Greenslade Collection. (Notes and picture by Thomas B. Greenslade, Jr., Kenyon College) 